\title{
Training Blind People in the Use of Graphical User Interfaces
}

\author{
Gerhard Weber $^{1,2}$, Helen Petrie ${ }^{3}$, Dirk Kochanek ${ }^{1}$, Sarah Morley 3 \\ ${ }^{1}$ Universität Stuttgart, Institut für Informatik, Breitwiesenstr. 20-22, 70565 Stuttgart, \\ F.R. Germany \\ 2 F.H. Papenmeier GmbH\&Co., KG, Talweg 2, 58239 Schwerte, F.R. Germany \\ 3 Psychology Division, University of Hertfordshire, Hatfield AL10 9AB, Hertfordshire, \\ U.K.
}

\begin{abstract}
The need for training of blind people to use graphical user interfaces has arisen since the first access systems became available. Two different approaches on the basis of PC-based tutorial systems are described and their benefits for the blind user are investigated.
\end{abstract}

\section{Introduction}

Most new applications like automatic teller machines, word processors, or database retrieval software are designed with a graphical user interface. Still, text-based applications are an alternative, but it is difficult to avoid MS Windows, $X$ Windows or some other graphical user interface (GUI).

For blind users several solutions are developed with a different design of the nonvisual user interface. Speech-based, Braille-based, or integrated systems offer access to MS Windows or $\mathrm{X}$ Windows with differences in speed of interaction and amount of information being accessible [3].

Tutorial systems have since long been inaccessible to blind users (and still remain) because of the integrated use of text, graphics, pictures, animated scenes and video. In other words: a blind user of the on-line tutorial system which became available with Windows 3.1 cannot perceive the animation provided to explain various selection tasks. As a consequence the tutorial system is of no use to the blind user even if they could benefit most from a tutorial system describing the basic interaction methods used in MS Windows.

\section{Training Needs in GUIs}

The development of GUIs poses a number of problems for blind users. They must learn new concepts associated with the ideas underlying the philosophy of GUIs, new methods of interaction with a computer, perhaps the use of new hardware. Careful training is required in all these areas if blind users are to access GUI based software 
most efficiently. Information provided by blind users about such software can be used in two ways. Firstly, it can assist in the development of better interfaces for blind users. Secondly, it can assist in the development of appropriate training programs to train blind users in the use of GUI based applications and operating systems.

For example, in the GUIB project, in order to maximise the usability of the nonvisual user interface and to develop appropriate training programs, the first prototype screenreaders for GUIs have been evaluated by blind computer users in two different fashions. Firstly, a laboratory-based evaluation of the interfaces has been undertaken; this required training blind users in the use of the interface and allowed evaluation both of the interface and the training material used. Secondly, the prototype GUIB system was made available to experienced blind users of text-based applications on a permanent, work place oriented basis for longer term evaluation in a realistic setting of use. In both these evaluations, it has quickly been found that this user group needs to learn about the functionality of GUIs and applications in a manner similar to computer novices. Depending on their experience with speech or Braille output they expect either speech-based or Braille-based interaction objects within a GUI.

A recent survey among trainers of blind computer users [8] as well as results from project IRIS which introduced screen readers for MS Windows at the Marburg school for the blind [4] have identified a number of requirements for the requirements for the training of blind computer users in GUIs. These include:

- stepwise introduction of concepts from GUIs

- use of tactile graphics and models

- development of concrete models and metaphors of GUIs

- development of diagnostic tasks which allow identification of specific conceptual problems of the user

GUIs contain many new concepts for blind users. For sighted persons some of the new concepts such as windows, dragging icons have intuitive meaning, but this is less likely for blind users. Therefore it may be helpful to introduce the concepts of GUIs in a meaningful progress. The notion of a "training wheels" version of the interface, where some of the functionality is blocked $[1,2]$ which has been used in training sighted users in the use of complex new interfaces is appropriate here.

\section{Tactile Graphical Images}

Permanent tactile graphics can be successfully used in the introduction of GUIs to blind users, although clearly they have little role in the normal use of GUIs. For interactive access to wordprocessors, spreadsheet programs, and other GUI-based programs, the current technology of tactile graphical output devices cannot be used efficiently. However, paper-based, solid or layered models are suitable. A teacher can progressively introduce elements of the tactile model and explain them with verbal presentations. A layered, solid model has the flexibility to convey dynamic changes such as window popping up or selection of a menu item.

Pictorial or graphical information can be conveyed as tactile picture or as a written or spoken description. A combination of a tactile picture with such 
explanation would be a better solution. For this purpose the program EXPLAIN was developed that allows an interactive exploration of tactile images accompanied by descriptions.

From a technical point of view such a combination makes some form of synchronisation through pointing by fingers or a stylus necessary. Several systems have been developed using different pointing devices:

- touch-sensitive screen (infrared technology, [9]),

- paper-based tactile graphics with touch-sensitive pad (pressure sensitive polymers, [7]),

- touch-sensitive interactive tactile display (electromagnetic induction, [5]), and

- paper-based tactile graphics with stylus (electromagnetic induction, [6] ).

In any of these systems explanations are linked to a specific area or region of the image which are selected by pointing or by gestural input. Figure 1 shows the basic configuration of program EXPLAIN which is used in project GUIB to aid introduction of GUIs.

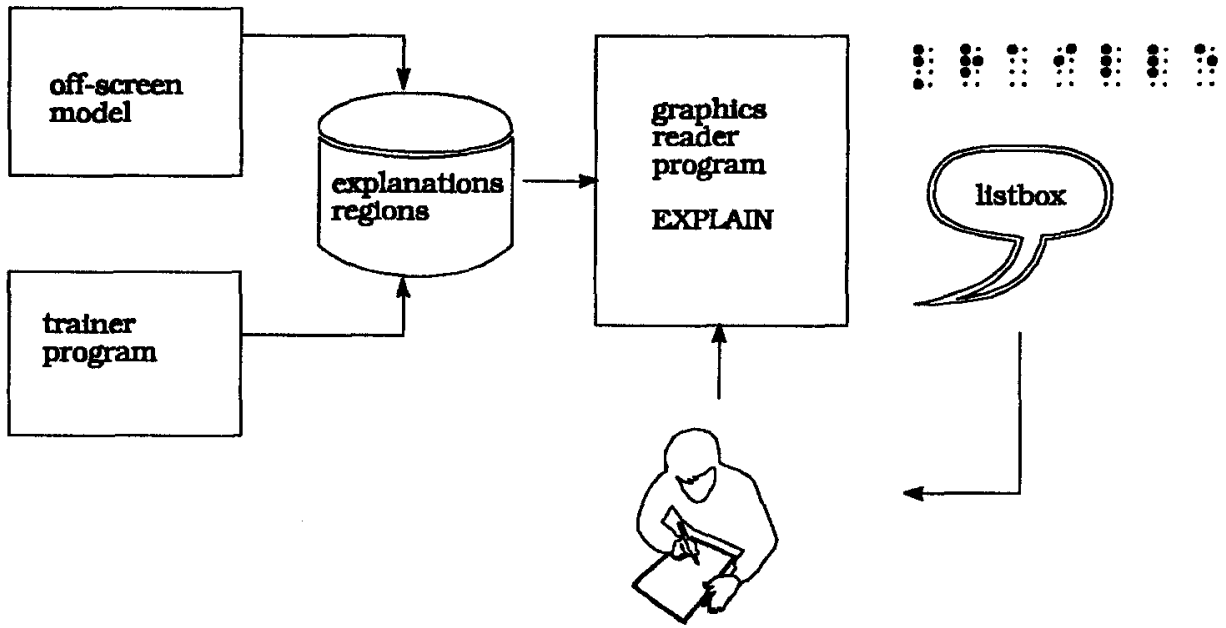

Fig. 1. Configuration of EXPLAIN

The pad has an touch sensitive area at least the size of a standard Braille page and a resolution of $120 * 120$ points. Speech output can be either done by a (external) speech synthesiser or using a standard sound card with text-to-speech software.

EXPLAIN consists of two components. The first component is used by an instructor to specify regions on an image and links explanations to this region. Explanations can be structured in levels allowing the trainee to quickly explore the image or get a more detailed explanation of a point of interest. The program facilitates creating of descriptions on the fly as well as loading them form external files.

The second component is used by a trainee who wants to explore an image. After placing the image on the touch pad the user has to identify the image to the program (by name or id number). This will cause the program to load the correct area definition and descriptions. Now the trainee can explore the image. Pressing on a 
spot will start the linked explanation of the specified level to be spoken. The user can change the explanation level or can repeat it.

Such a system requires a training phase during which explanations are introduced. Figure 1 shows the configuration for the interactive system. The user places a tactile graphics on a touch-sensitive pad and identifies it to the reader program. Through pointing by a stylus or by the fingers an explanation can be retrieved. Output is generated on a Braille display or by speech synthesis. To avoid time consuming preparation of the graphics a snapshot of screen contents can be generated and printed on a Braille printer. Instead of an extensive training phase the contents of the OSM of a particular screen reader can be used as the basis for the generation of verbal explanations.

\section{Interactive tutorial system}

After the basic concepts of GUIs have been introduced to the user a concrete model application should be used but under strict control of a tutorial program. Such an application introduces the basic interaction objects by using a screen reader with Braille or speech output. The drill\&practice tutorial system WINTRAIN for blind users has been developed in project GUIB for this purpose. WINTRAIN appears to the user as a simple text editor.

WINTRAIN is independent of a particular screen reader, nevertheless if required speech output through the Monologue text-speech system can be enabled. In this way the filtering within MS Windows is of dual use: for spoken feedback and for monitoring the completion of tasks. Figure 2 shows this architecture, those parts which are grayed are standard for a screen reader.

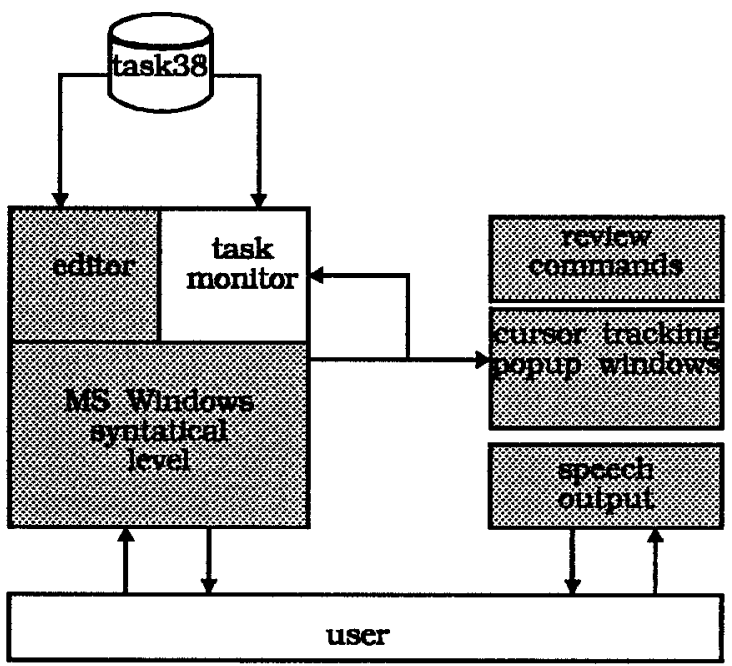

Fig. 2. Dual use of filters for WINTRAIN 
The built-in screen reader (with limited facilities for review) generates task descriptions and explains items as well as verbalises windows, menus, buttons, etc. Figures 3 and 4 show a typical scenario in WINTRAIN.

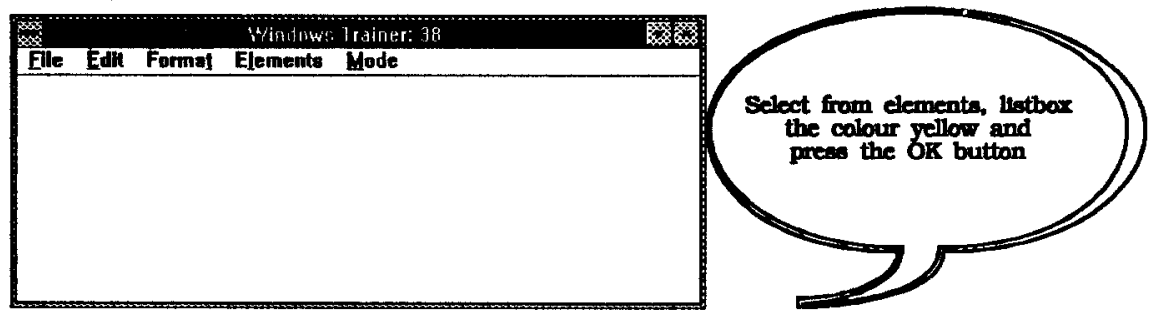

Fig. 3. A task is presented to the user

The user is asked to complete task 38 "select from elements listbox the colour yellow and press the OK button". After pressing "Alt" the menu options are verbalised. As usual in MS Windows the option "Elements" can be selected through mouse, cursor keys or the 'L'-key. A submenu opens with various options including "listbox". Finally the listbox as shown in Figure 4 is presented.

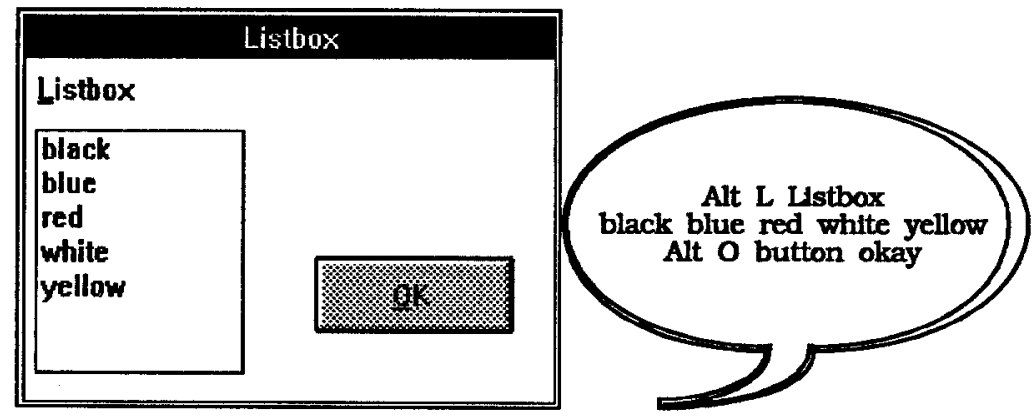

Fig. 4. The user opens a dialogue box

As soon as the user has selected in the listbox the option "yellow" the screen reader announces the closing of the dialogue box, the task monitor announces the successful completion of the task and presents the next task. A tutorial consists of a list of tasks specified in a configuration file. If $50 \%$ of the tutorials are successfully completed the next tutorial is started. The following common interaction methods are addressed by the more then 40 tasks:

- menu selection (main menu, popup menu),

- common interaction objects in dialogue boxes (button, radiobutton, checkbox, edit field, listbox, combobox),

- open a file by name and path,

- print a file,

- format a character (font, size, colour, etc.),

- find respectively replace a string,

- read text and move the caret to a specified position within a line of text, and

- resize respectively move the window of WINTRAIN. 
This set of tasks reflects the common interaction methods found e.g. in word processors, spreadsheet programs, and other standard MS windows programs. After completing all tutorials the program can be finished including the Windows environment. Thus the user has full control over interaction while working with an Windows application and returns from there to DOS.

Since the training program - unlike to more advanced tutorial systems for GUIs avoids any animated scenes and graphics any screen reader for MS Windows provides sufficient access to it. An self-explanatory facility is provided further on to help the user in solving his task.

Explanations for beginners should cover

- a short description of the interation method

- a verbal description of the layout of the current window res. the relation among interaction objects

- a verbal description of the layout of windows

By choosing an application from a well known domain (text processing) the blind user is not learning about the application specific operations. Instead they can transfer this knowledge about interaction into the domain of GUIs. While the interaction objects also appear in DOS-based application programs the specific behaviour inside MS Windows can be trained

Several forms of evaluation of these training programs are being undertaken as part of the GUIB Project. For both the EXPLAIN and the WINTRAIN programs, users are given a test of their knowledge of the use of GUI software to measure the usefulness and acceptability on these programs. Users who participate in the WINTRAIN tutorial program are also given a test of their knowledge of the use of GUI software to objectively assess how much they have learnt from the program.

If the above requirements can be included during run-time of the screen reader (and not the tutorial software) a more powerful context-sensitive passive help system can be created (on the basis of the OSM and filtering the user interface) then was originally anticipated.

The limits to this approach are lack of application specific information. In order to overcome this deficit plan recognition together with a model of the user's task can be used. By interpreting such a task model the completion of a particular series of interactions can be initiated automatically as it would be required for an active help system.

\section{Conclusions}

The benefits of a GUIs will only become available also to blind users if screen readers are designed well enough for successful non-visual interaction. In order to enable blind users to participate in this process they need prototypical screen readers and assistance while learning to interact with an application. This assistance can be provided more easily through flexible tutorial systems. The final paper will refer to feedback given by blind users trying to learn more MS Windows using the presented tutorial system. 


\section{Acknowledgements}

This work has been supported by the GUIB consortium, a pilot research project of the EEC program "Technology Initiative for the Disabled and Elderly" (TIDE).

\section{References}

[1] Carroll, J.M. and Carrithers, C. (1984). Training wheels in a user interface. Communications of the ACM, 27, pp. 800 - 806

[2] Carroll, J.M., Kellog, W.A. and Rosson, M.B. (1991). The task-artifact cycle. In J.M.Carroll (Ed.) Designing interaction: psychology at the human-computer interface. Cambridge: Cambridge University Press

[3] Mynatt, E.; Weber, G. (1994). Nonvisual presentation of graphical user interfaces: contrasting two approaches, in Proceedings of CHI'94, Boston May 26-28, 1994, New York: ACM

[4] Kalina, U. (1993). personal communication, Dec. 14th, 1993

[5] Kochanek, D. (1992). A hypertext system for blind newspaper readers, in Zagler, W. (ed.) Proceedings of the 3rd International Conference on Computers for Handicapped People, Wien: Oldenbourg

[6] Lötzsch, J.: (1993). Audiotaktiler Dialog über Graphiken und Diagrammen insbesondere für Blinde und Sehbehinderte (Audiotactile dialog on graphics and diagrams for blind and visually impaired), in Mehnert, D. (ed.) Elektronische Sprachsignalverarbeitung in der Rehabilitationstechnik, ISSN 0940-6832, pp.153-156

[7] Parkes (1991). Nomad: enabling access to graphics and text-based information for blind and visually impaired and other disability groups, in Proceedings of the World Congress on Technology for People with Disabilities, Washington DC, December 1-5, 1991, pp. 689-716

[8] Petrie, H. (1992). Report on training aspects for blind users in graphics-based environments. Report to the CEC for TIDE Pilot Action Project 103: Graphical User Interfaces for Blind Persons. London: Royal National Institut for the Blind

[9] Weber, G. (1987). Gestures as a means for the blind to interact with a computer, in Bullinger, H.-J. (ed.) Human-Computer Interaction INTERACT'87, Amsterdam: North Holland, pp. 593-595 